\title{
Nodal Integration Technique in Meshless Method
}

\author{
Ahmed MJIDILA ${ }^{1}$, Salah Eddine JALAL ${ }^{2}$, Lahbib BOUSSHINE ${ }^{3}$, \\ Zakaria EL LASKAOUI ${ }^{4}$ \\ 1,2,3,4 (Ain Chock Hassan II University, NHSEM, Oasis, Eljadida road, PB8118 Casablanca \\ Laboratory of Technologies of Constructions and the Industrial Systems)
}

\begin{abstract}
In this paper an integration technique is resented. In 1985, G.R. Lui and coworkers used this technique to integrate weak form formulation over line support in $1 D$ case or over support-Domain in $2 D$ case in Finite Element and Meshless methods. Present paper shows the results calculus by a Fortran Code, written in our laboratory, in both cases: regular and irregular nodal distribution.

Keywords: Node, Gauss quadrature, Nodal Integration, Mesh-less method, Stiffness matrix.
\end{abstract}

\section{Introduction}

Numerical integration plays an important role in the process of a meshfree weak form method. Gauss integration scheme is commonly used for integration of weak form methods. As we know, the finite element method uses Gauss quadrature in each element to integrate the weak form. For most of meshfree methods based on Galerkin weak form, error will be involved in the process of numerical integration due to the complexity involved in Gauss integration (Dolbow and Belytschko, 1999). Then some types of nodal integration schemes have been developed to perform the numerical integration. The core idea of a nodal integration scheme is to use nodes as the integration sampling points.

\section{Discretized System Equations}

Consider a two-dimensional solid problem defined in domain $\Omega$ bounded by $\Gamma\left(\Gamma=\Gamma_{u}+\Gamma_{t}\right)$, the governing equations of this problem can be expressed as follows (Liu, 2002).

- Equilibrium equation:

$$
L^{T} \sigma+b=0 \quad \text { in } \Omega
$$

- Natural boundary condition:

$$
\sigma \cdot n=t \quad \text { on } \Gamma_{t}
$$

- $\quad$ Essential boundary condition:

$$
u=\bar{u} \quad \text { on } \Gamma_{u}
$$

Where $L^{T}=\left[\begin{array}{ccc}\frac{\partial}{\partial x} & 0 & \frac{\partial}{\partial y} \\ 0 & \frac{\partial}{\partial y} & \frac{\partial}{\partial x}\end{array}\right]$ is differential operator $; \sigma^{T}=\left\{\sigma_{x x}, \sigma_{y y}, \sigma_{x y}\right\}$ is the stress vector; $u^{T}=\{u, v\}$ is the displacement vector; $b^{T}=\left\{b_{x}, b_{y}\right\}$ is the body force vector, $\bar{t}$ is the prescribed traction on the natural boundaries, $\mathrm{u}$ is the prescribed displacement on the essential boundaries, and $\mathbf{n}$ is the vector of unit outward normal at a point on the natural boundary.

The unconstrained Galerkin weak form of Equation 1 is as follows (Liu, 2002),

$$
\int_{\Omega}(\mathbf{L} \boldsymbol{\delta} \mathbf{u})^{T}(\mathbf{D L} \mathbf{u}) d \Omega-\int_{\Omega} \boldsymbol{\delta} \mathbf{u}^{T} \mathbf{b} d \Omega-\int_{\Gamma_{t}} \boldsymbol{\delta} \mathbf{u}^{T} \mathbf{t} d \Gamma=0
$$

For linear elasticity, the material matrix D is expressed as follows, 


$$
\begin{gathered}
D=\frac{E}{1-\nu^{2}}\left[\begin{array}{ccc}
1 & \nu & 0 \\
\nu & 1 & 0 \\
0 & 0 & \frac{1-\nu}{2}
\end{array}\right] \quad \text { for plane stress problem } \\
D=\frac{E(1-\nu)}{(1+\nu)(1-2 \nu)}\left[\begin{array}{ccc}
1 & \frac{\nu}{1-\nu} & 0 \\
\frac{\nu}{1-\nu} & 1 & 0 \\
0 & 0 & \frac{1-2 \nu}{2(1-\nu)}
\end{array}\right] \quad \text { for plane strain problem }
\end{gathered}
$$

where E is Young's modulus and v is Poisson's ratio. Substituting the RPIM approximation equation [1],

$$
\mathbf{u}^{h}(x)=\left[\mathbf{R}^{T}(x) \mathbf{S}_{a}+\mathbf{P}^{T}(x) \mathbf{S}_{b}\right] \mathbf{U}_{s}=\boldsymbol{\Phi}(x) \mathbf{U}_{s}
$$

into equation 4 we obtain,

$$
K d=f
$$

where $\mathrm{d}$ is the vector of nodal displacement at all the unconstrained nodes and

$$
\begin{gathered}
\mathbf{K}_{i j}=\int_{\Omega} \mathbf{B}_{i}^{T} \mathbf{D} \mathbf{B}_{j} d \Omega \\
\mathbf{f}_{i}=\int_{\Gamma_{t}} \varphi_{i} \overline{\mathbf{t}} d \Gamma+\int_{\Omega} \varphi_{i} \mathbf{b} d \Omega
\end{gathered}
$$

In witch

$$
\mathbf{B}_{i}=\left[\begin{array}{cc}
\phi_{i, x} & 0 \\
0 & \phi_{i, y} \\
\phi_{i, y} & \phi_{i, x}
\end{array}\right]
$$

To de.ne the global stiffness matrix integration calculus becomes necessary, see equation 9. Usually, Gauss points method is used. The nodal integration method can be an alternative technique to carry out the integration calculus and it's more adapted to meshless approach, as it will be presented in following.

We have an integral

\section{Nodal Integration Scheme}

$$
I=\int_{\Omega} f(x) d \Omega
$$

where $\square(\square)$ is an integrable function, which is, for example, a component of matrix $B_{i}^{T} D B_{j}$ given in Equation 9; $\Omega$ is the domain of the problem, that is represented by a set of $\square \square$ nodes distributed in the problem domain. In a nodal integration scheme, the domain $\Omega$ is divided into a set of non-overlapping sub-domains $\Omega_{\mathrm{i}} \square\left(\square=1 \square \square 2 \square \square \square \square \square \square \square\right.$ ), each of them includes a node, and $\Omega=\sum_{i=1}^{N} \Omega_{i} \square \square$ Then the integrated, Equation 12, can then be expressed as

$$
I=\sum_{i=1}^{N} \int_{\Omega_{i}} f(x) d \Omega_{i}
$$

In a meshfree method based on weak-form, a background mesh is needed for the implementation of numerical integration. For the present method, a background mesh is used for constructing the nodal integration domain for each node. The background mesh is not used for shape function construction which is constructed using a same set of nodes located in a local support domain. The independence of mesh from shape function construction has many advantages including the improvement in accuracy, which will be observed later in examples. This fact has also been found in many other existing works (Belytschko and al., 1994; Atluri and Zhu, 1998; Chen et al., 2001). It is very clear that one does not have to use mesh for shape function construction. In the present work, the mesh is also only used for integration purpose. The question now is how 
to evaluate $\int_{\Omega_{i}} f(x) d \Omega_{i}$ over the nodal integration domain $\Omega$ i. Here a novel and simple approach based on the Taylor series extension is presented.

The basic idea of this approach is to extend the integral function to some terms of Taylor series, and the integration will be approximately performed on these terms. Note that the integrand $f(x)$ is required to be differentiable within the integration domain when it is extended to be terms of Taylor series. Therefore, RPIM shape functions are constructed using the same set of nodes in each integration domain. A shape function constructed is one-piece, and hence is differentiable to any order in the integration domain. Note that the discontinuity will occur on the interfaces of the integration domains, and hence causes the non-conformability, which is omitted in this work, as it is controlled by the use of RBF shape functions with proper shape parameters (Liu, 2002). Note that this kind of non-conformability exists for all the meshfree methods based on weak-form and nodal integration even the ones using MLS shape functions, unless strain smoothing technique is used (Chen and al., 2001; Liu and al., 2005a; Liu and Zhang, 2006).

For comparison, the EFG method based on nodal integration is also coded, in which shape functions are obtained using the MLS method (Belytschko and al., 1994). For convenience, this method is named as NI-MLS. It is known that the MLS shape functions can be constructed to satisfy the compatibility condition and the continuity of the field function approximation is ensured (Liu, 2002).

\subsection{For 1D problems}

\section{Formulation Of Nodal Integration}

To explain the method more clearly, the formulations for one-dimensional problems will be first presented. Based on Taylor series extension, a continuous function $\square(\square)$ can be approximated in the vicinity of a point $\square_{\square}$ as follows [2]

$$
f(x) \approx f\left(x_{0}\right)+f^{\prime}\left(x_{0}\right) x+\frac{f^{\prime \prime}\left(x_{0}\right)}{2 !} x^{2}
$$

The 3rd order and above are truncated.

The integration for the function $\square(\square)$ in the domain $\left(x_{1} \leq x \leq x_{2}\right)$ can then be evaluated as:

$$
\begin{aligned}
\int_{x_{1}}^{x_{2}} f(x) d x & \approx \int_{x_{1}}^{x_{2}}\left(f\left(x_{0}\right)+f^{\prime}\left(x_{0}\right) x+\frac{f^{\prime \prime}\left(x_{0}\right)}{2 !} x^{2}\right) d x \\
& =\int_{x_{1}}^{x_{2}} f\left(x_{0}\right) d x+\int_{x_{1}}^{x_{2}} f^{\prime}\left(x_{0}\right) x d x+\int_{x_{1}}^{x_{2}} \frac{f^{\prime \prime}\left(x_{0}\right)}{2 !} x^{2} d x \\
& =f\left(x_{0}\right)\left(x_{2}-x_{1}\right)+\frac{1}{2} f^{\prime}\left(x_{0}\right)\left(x_{2}^{2}-x_{1}^{2}\right)+\frac{1}{6} f^{\prime \prime}\left(x_{0}\right)\left(x_{2}^{3}-x_{1}^{3}\right)
\end{aligned}
$$

Considering now a one-dimensional problem, the problem domain is presented by a set of nodes, as shown in Figure 1and Figure 2 . The integrand of $\mathrm{f}(\mathrm{x})$ is now a component of the matrix $B_{i}{ }^{T} D B_{j}$ (see Equation 9). When the field-nodes are regularly distributed, by using Equation (15), the numerical integration for the $\mathrm{i}^{\text {th }}$ node can be performed as follows.

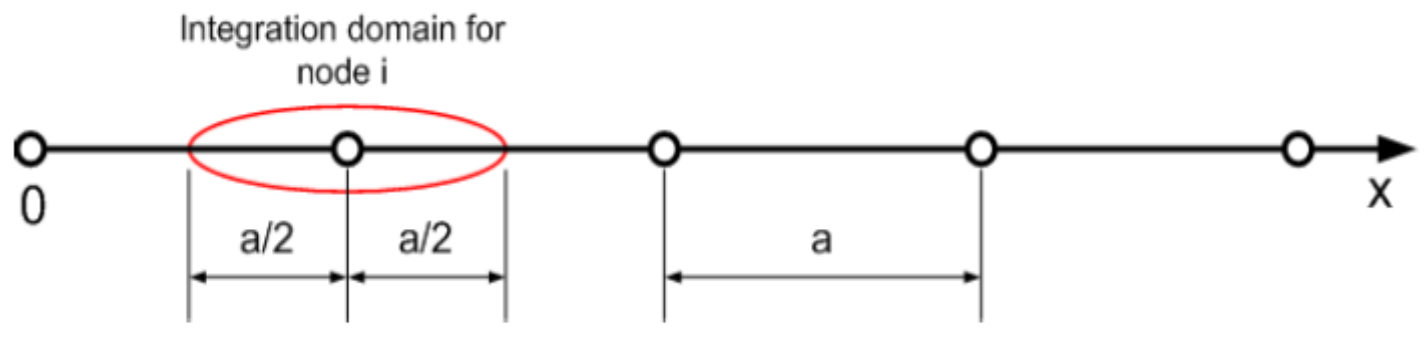

Figure 1 Integration domain with regular nodal distribution. 


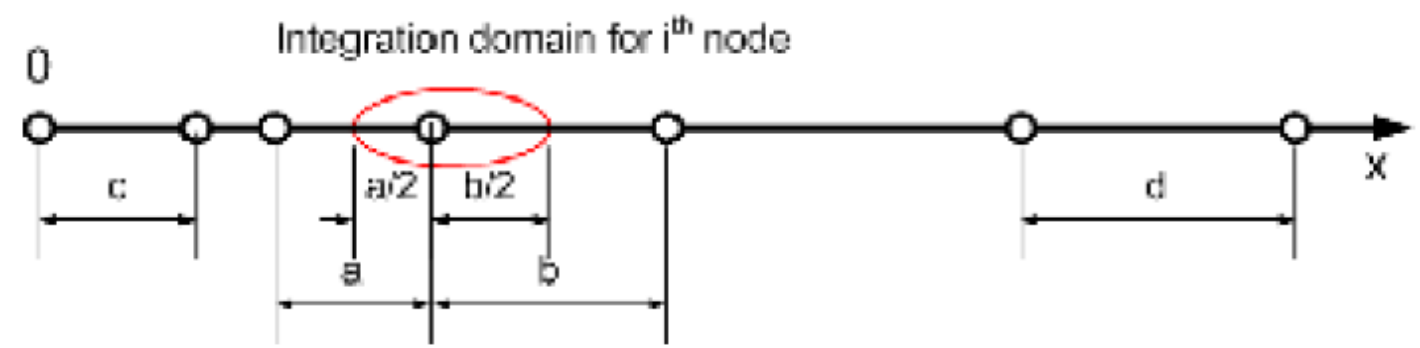

Figure 2 Integration domain with irregular nodal distribution

Case 1: regularly distributed nodes

\section{For an internal node}

For an internal node, the integration can be applied as:

$$
\begin{aligned}
\int_{-\frac{a}{2}}^{\frac{a}{2}} f(x) d x= & f\left(x_{i}\right)\left(\frac{a}{2}+\frac{a}{2}\right)+\frac{1}{2} f^{\prime}\left(x_{i}\right)\left[\left(\frac{a}{2}\right)^{2}-\left(-\frac{a}{2}\right)^{2}\right]+ \\
& \frac{1}{6} f^{\prime \prime}\left(x_{i}\right)\left[\left(\frac{a}{2}\right)^{3}-\left(-\frac{a}{2}\right)^{3}\right] \\
= & f\left(x_{i}\right) a+\frac{1}{24} f^{\prime \prime}\left(x_{i}\right) a^{3}
\end{aligned}
$$

where a is the nodal spacing as shown in Figure 1.

For the node at the left end

For the node located at the left end, the integration will as:

$$
\begin{aligned}
\int_{0}^{\frac{a}{2}} f(x) d x & =f\left(x_{i}\right)\left(\frac{a}{2}-0\right)+\frac{1}{2} f^{\prime}\left(x_{i}\right)\left[\left(\frac{a}{2}\right)^{2}-0\right]+\frac{1}{6} f^{\prime \prime}\left(x_{i}\right)\left[\left(\frac{a}{2}\right)^{3}-0\right] \\
& =\frac{1}{2} f\left(x_{i}\right) a+\frac{1}{8} f^{\prime}\left(x_{i}\right) a^{2}+\frac{1}{48} f^{\prime \prime}\left(x_{i}\right) a^{3}
\end{aligned}
$$

For the end at the right end

For the node located at the right end of the 1D domain,

$$
\begin{aligned}
\int_{-\frac{a}{2}}^{0} f(x) d x= & f\left(x_{i}\right)\left(0+\frac{a}{2}\right)+\frac{1}{2} f^{\prime}\left(x_{i}\right)\left[0-\left(\frac{a}{2}\right)^{2}\right]+ \\
& \frac{1}{6} f^{\prime \prime}\left(x_{i}\right)\left[0-\left(-\frac{a}{2}\right)^{3}\right] \\
= & \frac{1}{2} f\left(x_{i}\right) a-\frac{1}{8} f^{\prime}\left(x_{i}\right) a^{2}+\frac{1}{48} f^{\prime \prime}\left(x_{i}\right) a^{3}
\end{aligned}
$$

Case 2: irregularly distributed nodes

When the field nodes are irregularly distributed, Equations (16), (17) and (18) can be expressed as follows.

For an internal node:

For internal node, the integration will be applied as:

$$
\begin{aligned}
\int_{-\frac{a}{2}}^{\frac{b}{2}} f(x) d x= & f\left(x_{i}\right)\left(\frac{b}{2}+\frac{a}{2}\right)+\frac{1}{2} f^{\prime}\left(x_{i}\right)\left[\left(\frac{b}{2}\right)^{2}-\left(-\frac{a}{2}\right)^{2}\right]+ \\
& \frac{1}{6} f^{\prime \prime}\left(x_{i}\right)\left[\left(\frac{b}{2}\right)^{3}-\left(-\frac{a}{2}\right)^{3}\right] \\
= & \frac{1}{2} f\left(x_{i}\right)(a+b)+\frac{1}{8} f^{\prime}\left(x_{i}\right)\left(b^{2}-a^{2}\right)+\frac{1}{48} f^{\prime \prime}\left(x_{i}\right)\left(a^{3}+b^{3}\right)
\end{aligned}
$$




\section{For the node at the left end}

For the node located at the left end, the integration will as:

$$
\begin{aligned}
\int_{0}^{\frac{c}{2}} f(x) d x= & f\left(x_{i}\right)\left(\frac{c}{2}-0\right)+\frac{1}{2} f^{\prime}\left(x_{i}\right)\left[\left(\frac{c}{2}\right)^{2}-0\right]+ \\
& \frac{1}{6} f^{\prime \prime}\left(x_{i}\right)\left[\left(\frac{c}{2}\right)^{3}-0\right] \\
= & \frac{1}{2} f\left(x_{i}\right) c+\frac{1}{8} f^{\prime}\left(x_{i}\right) c^{2}+\frac{1}{48} f^{\prime \prime}\left(x_{i}\right) c^{3}
\end{aligned}
$$

For the end at the right end

For the node located at the right end of the 1D domain,

$$
\begin{aligned}
\int_{-\frac{d}{2}}^{0} f(x) d x & =f\left(x_{i}\right)\left(0+\frac{d}{2}\right)+\frac{1}{2} f^{\prime}\left(x_{i}\right)\left[0-\left(\frac{d}{2}\right)^{2}\right]+\frac{1}{6} f^{\prime \prime}\left(x_{i}\right)\left[0-\left(-\frac{d}{2}\right)^{3}\right] \\
& =\frac{1}{2} f\left(x_{i}\right) d-\frac{1}{8} f^{\prime}\left(x_{i}\right) d^{2}+\frac{1}{48} f^{\prime \prime}\left(x_{i}\right) d^{3}
\end{aligned}
$$

where $a, b, c$ and $d$ are nodal spacing for the irregularly distributed nodes as shown in Figure2.

4.2. Numerical example

We calculate $I=\int 3 x^{2} d x$ in domain [0,10]. The domain is divided in eleven nodes in which the integrals are

calculated as shows table 1. Figure 3 shows the analytical and approximated results which coincide very good. These results have been used to plot figure 3 .

\begin{tabular}{|l|l|l|}
\hline Node & Analytical value & Value by Taylor's series \\
\hline 0 & $\int_{0}^{0.5} 3 x^{2} d x: 0.125$ & $\int_{0}^{0.5} 3 x^{2} d x=\frac{1}{2}\left(3 * 0^{2}\right)-\frac{1}{8}(6 * 0) * 1^{2}+\frac{1}{48}(6) * 1^{3}=0.125$ \\
\hline 1 & $\int_{0.5}^{1.5} 3 x^{2} d x=3.25$ & $\int_{-0.5}^{0.5} 3 x^{2} d x=\left(3 * 1^{2}\right)+\frac{1}{24}(6) * 1^{3}=3.25$ \\
\hline 2 & $\int_{1.5}^{2.5} 3 x^{2} d x=12.25$ & $\int_{-0.5}^{0.5} 3 x^{2} d x=\left(3 * 2^{2}\right)+\frac{1}{24}(6) * 1^{3}=12.25$ \\
\hline 3 & $\int_{2.5}^{3.5} 3 x^{2} d x=27.25$ & $\int_{-0.5}^{0.5} 3 x^{2} d x=\left(3 * 3^{2}\right)+\frac{1}{24}(6) * 1^{3}=27.25$ \\
\hline 4 & $\int_{3.5}^{4.5} 3 x^{2} d x=48.25$ & $\int_{-0.5}^{0.5} 3 x^{2} d x=\left(3 * 4^{2}\right)+\frac{1}{24}(6) * 1^{3}=48.25$ \\
\hline 5 & $\int_{4.5}^{5.5} 3 x^{2} d x=75.25$ & $\int_{-0.5}^{0.5} 3 x^{2} d x=\left(3 * 5^{2}\right)+\frac{1}{24}(6) * 1^{3}=75.25$ \\
\hline 6 & $\int_{5.5}^{6.5} 3 x^{2} d x=108.25$ & $\int_{-0.5}^{0.5} 3 x^{2} d x=\left(3 * 6^{2}\right)+\frac{1}{24}(6) * 1^{3}=108.25$ \\
\hline 7 & $\int_{6.5}^{7.5} 3 x^{2} d x=147.25$ & $\int_{-0.5}^{0.5} 3 x^{2} d x=\left(3 * 7^{2}\right)+\frac{1}{24}(6) * 1^{3}=147.25$ \\
\hline 8 & $\int_{7.5}^{8.5} 3 x^{2} d x=192.25$ & $\int_{-0.5}^{0.5} 3 x^{2} d x=\left(3 * 8^{2}\right)+\frac{1}{24}(6) * 1^{3}=192.25$ \\
\hline 9 & $\int_{8.5}^{9.5} 3 x^{2} d x=243.25$ & $\int_{-0.5}^{0.5} 3 x^{2} d x=\left(3 * 9^{2}\right)+\frac{1}{24}(6) * 1^{3}=243.25$ \\
\hline 10 & $\int_{9.5}^{10} 3 x^{2} d x=142.63$ & $\int_{-0.5}^{0} 3 x^{2} d x=\frac{1}{2}\left(3 * 10^{2}\right)-\frac{1}{8}(6 * 10) * 1^{2}+\frac{1}{48}(6) * 1^{3}=142.63$ \\
\hline
\end{tabular}

Table 1 Analytical and approximated integral values for $1 D$ case 


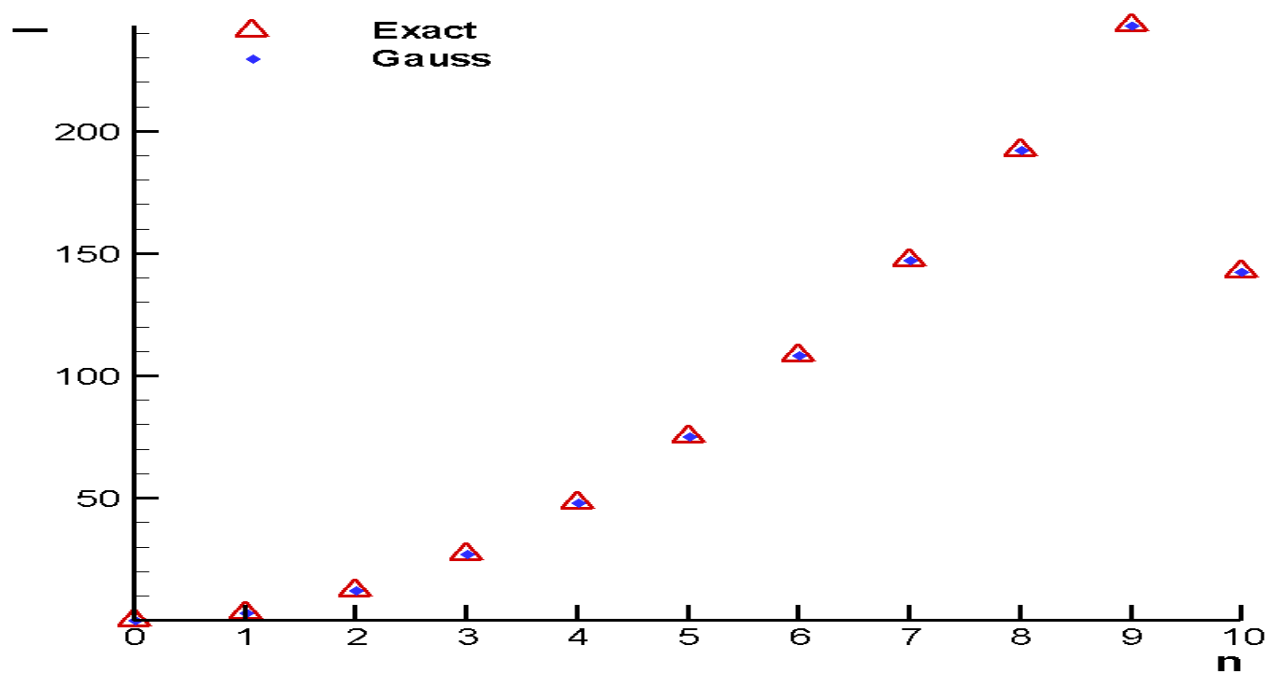

Figure 3 Analytical and approximated integral values 1D-case

\section{3. for 2D problems}

Applying Taylor series extension, a two-dimensional (2D) continuous function $\mathrm{f}(\mathrm{x}, \mathrm{y})$ can be approximated in the vicinity of point $\left(\mathrm{x}_{0}, \mathrm{y}_{0}\right)$ as follows,

$$
f(x, y) \approx f\left(x_{0}, y_{0}\right)+\left(x \frac{\partial}{\partial x}+y \frac{\partial}{\partial y}\right) f\left(x_{0}, y_{0}\right)+\frac{1}{2 !}\left(x \frac{\partial}{\partial x}+y \frac{\partial}{\partial y}\right)^{2} f\left(x_{0}, y_{0}\right)
$$

The integration for function $\square\left(\square \square \square \square\right.$ ) over the nodal integration domain $\Omega_{\square} \square$ can be expressed as,

$$
\begin{aligned}
\iint_{\Omega_{i}} f(x, y) d \Omega \approx & \iint_{\Omega_{i}}\left(f\left(x_{0}, y_{0}\right)+\left(x \frac{\partial}{\partial x}+y \frac{\partial}{\partial y}\right) f\left(x_{0}, y_{0}\right)+\frac{1}{2 !}\left(x \frac{\partial}{\partial x}+y \frac{\partial}{\partial y}\right)^{2} f\left(x_{0}, y_{0}\right)\right) \\
= & f\left(x_{0}, y_{0}\right) \iint_{\Omega_{i}} d \Omega+f_{, x}\left(x_{0}, y_{0}\right) \iint_{\Omega_{i}} x d \Omega+f_{, y}\left(x_{0}, y_{0}\right) \iint_{\Omega_{i}} y d \Omega \\
& +\frac{1}{2} f_{, x x}\left(x_{0}, y_{0}\right) \iint_{\Omega_{i}} x^{2} d \Omega+f_{, x y}\left(x_{0}, y_{0}\right) \iint_{\Omega_{i}} x y d \Omega+ \\
& \frac{1}{2} f_{, y y}\left(x_{0}, y_{0}\right) \iint_{\Omega_{i}} y^{2} d \Omega \\
= & f\left(x_{0}, y_{0}\right) A_{i}+f_{, x}\left(x_{0}, y_{0}\right) M_{y_{i}}+f_{, y}\left(x_{0}, y_{0}\right) M_{x_{i}}+\frac{1}{2} f_{, x x}\left(x_{0}, y_{0}\right) M_{y y_{i}} \\
& +f_{, x y}\left(x_{0}, y_{0}\right) M_{x y_{i}}+\frac{1}{2} f_{, y y}\left(x_{0}, y_{0}\right) M_{x x_{i}}
\end{aligned}
$$

where :

$$
\begin{array}{rlrl}
A_{i} & =\iint_{\Omega_{i}} d \Omega, & & \text { Area of domain of } i^{t h} \text { node } \\
M_{x i} & =\iint_{\Omega_{i}} y d \Omega, & M_{y_{i}}=\iint_{\Omega_{i}} x d \Omega,
\end{array}
$$

the area moments of $1 \square \square \square$ order for the domain of the $\mathrm{i} \square \square \square$ node,

$$
M_{x x i}=\iint_{\Omega_{i}} y^{2} d \Omega, M_{y y i}=\iint_{\Omega_{i}} x^{2} d \Omega, M_{x y i}=\iint_{\Omega_{i}} x y d \Omega,
$$

the area moments of $2 \square \square \square$ order for the domain of the $\mathrm{i} \square \square \square$ node .

4.4. The integration along the boundary line

The integration for function $\square(\square \square \square \square)$ along the boundary line can be formulated as: 


$$
\begin{aligned}
\int_{\Gamma} f(x, y) d l= & \int_{\Gamma}\left(f\left(x_{0}, y_{0}\right)+\left(x \frac{\partial}{\partial x}+y \frac{\partial}{\partial y}\right) f\left(x_{0}, y_{0}\right)+\frac{1}{2 !}\left(x \frac{\partial}{\partial x}+y \frac{\partial}{\partial y}\right)^{2} f\left(x_{0}, y_{0}\right)\right) d l \\
= & f\left(x_{0}, y_{0}\right) \int_{\Gamma} d l+f_{, x}\left(x_{0}, y_{0}\right) \int_{\Gamma} x d l+f_{, y}\left(x_{0}, y_{0}\right) \int_{\Gamma} y d l \\
& +\frac{1}{2} f_{, x x}\left(x_{0}, y_{0}\right) \int_{\Gamma} x^{2} d l+f_{, x x}\left(x_{0}, y_{0}\right) \int_{\Gamma} x y d l+\frac{1}{2} f_{, y y}\left(x_{0}, y_{0}\right) \int_{\Gamma} y^{2} d l
\end{aligned}
$$

To apply the nodal integration technique, a background cell is needed to divide the problem domain into nodal integration domains, each of which includes a node.

When the nodes are regularly distributed, a rectangular domain can be used as the nodal integration domain $\square \square($ illustrated in Figure ), and the union of all the rectangles forms the problem domain. As shown in 5.1, when the nodes are irregularly distributed, a tessellation can always be generated automatically by joining the centroids of the triangles and the mid-edge points (Ferzige and Peric, 1999).

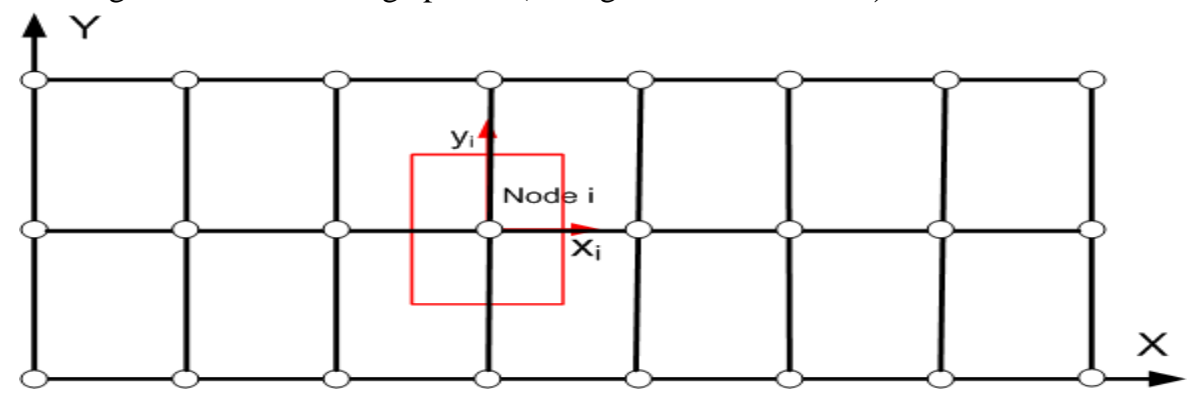

Figure 4 Background cells in case: Regular distribution of nodes in domain integration.

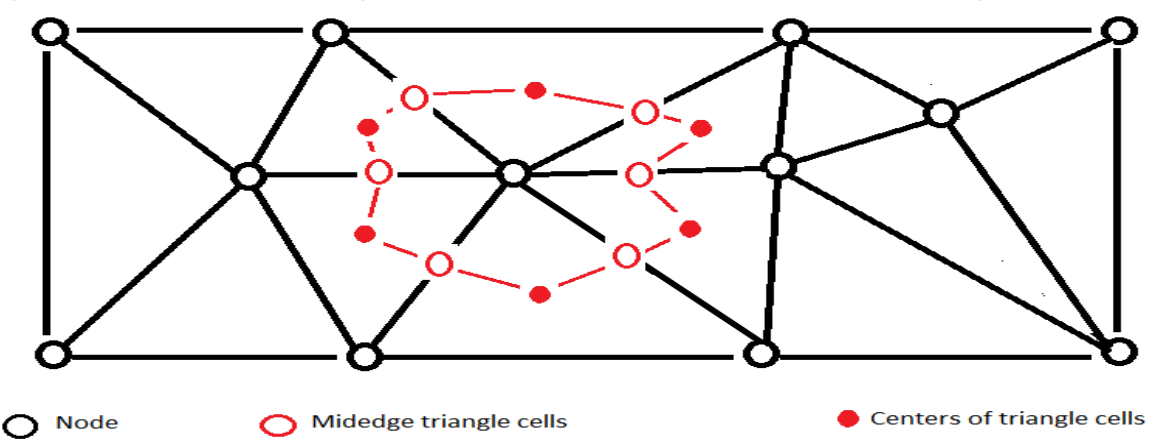

Figure 5 Background cells in case: Irregular distribution of nodes in domain integration

The area $\mathrm{A}_{\mathrm{i}}$, the moments $\mathrm{M}_{\mathrm{xi}}, \mathrm{M}_{\mathrm{yi}}, \mathrm{M}_{\mathrm{xxi}}, \mathrm{M}_{\mathrm{xyi}}$, and $\mathrm{M}_{\mathrm{yyi}}$ for the $\mathrm{i}^{\text {th }}$ field node can be calculated during the preprocess stage for later use in the numerical integration, because they depend on only the geometry of the nodal integration domain.

4.5. Numerical example : two-dimension case

Area test A is chosen, a $2 \times 2$ plate with 9 nodes distributed as shows figures 6 , regular (a) and irregular (b) manner.
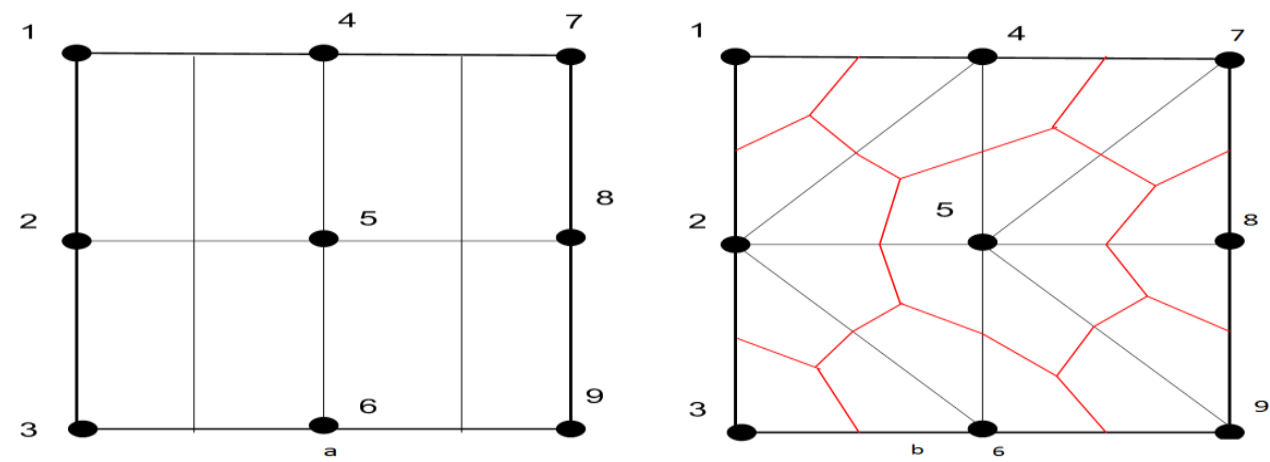

Figure 6 Area test plaque 2x2, with regular (a) and irregular (b) nodal distribution

As a benchmark test, the following integral is chosen $I=\int 2 x^{2} y^{2} d x d y \square$ over the test area A.

A Fortran code, written in our Laboratory, for each area calculates all necessary geometrical characteristics and evaluates the integral values in regular and irregular cases. The following table 2 gives the cumulus-results 
calculated by Maple, Analytical method and by present methods in both cases: regular and irregular nodal distribution. The following plot (figure 7) gives comparison between analytical and present methods in regular and irregular nodal distribution.

\begin{tabular}{|c|c|c|c|c|}
\hline Node & Maple & Analyt. & Reg. & Irreg. \\
\hline 1 & 0.128470 & 0,128470 & 0,166666 & 0.070400 \\
\hline 2 & 0.218748 & 0.218677 & 0.250000 & 0.218600 \\
\hline 3 & 0.222220 & 0.218677 & 0.250000 & 0.218600 \\
\hline 4 & 3.5625202 & 3.562419 & 3.666667 & 4.752256 \\
\hline 5 & 5.9097202 & 5.909641 & 6.000000 & 6.227656 \\
\hline 6 & 5.9999982 & 5.999918 & 6.083333 & 6.378456 \\
\hline 7 & 10.7534982 & 10.75339 & 10.91597 & 9.983253 \\
\hline 8 & 14.0937982 & 14.093667 & 14.33264 & 14.502775 \\
\hline 9 & 14.2222682 & 14.222139 & 14.49931 & 14.574774 \\
\hline
\end{tabular}

Table 2 Analytical and approximated integral values for 2D case,(by Maple, Analytical calculus, Taylor's series with regular nodal distribution, with irregular distribution)

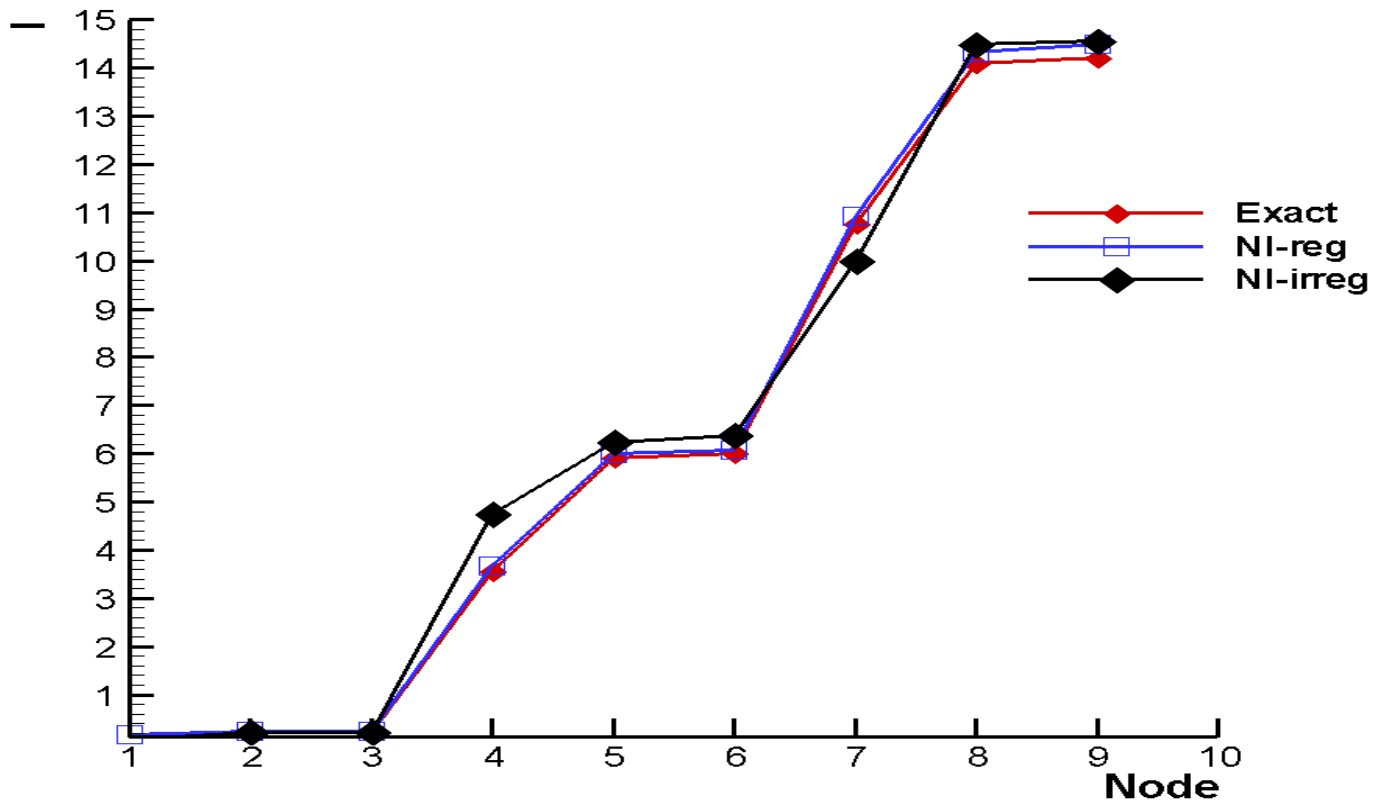

Figure 7 Integration Results : Red, Analytical - Blue, Present method with regular nodal distributionBlack, Present method with irregular nodal distribution.

\section{Discussion}

The results given by present integration method, named in this paper nodal integration, are very accurate. The error's calculation gives:

- For regular nodal distribution:

$$
e=\frac{\left|I_{e x}-I_{\text {code }}\right|}{I_{e x}} .100 \%=\frac{|14.222139-14.49931|}{14.222139} .100=1.94 \%
$$

- $\quad$ For irregular nodal distribution:

$$
e=\frac{\left|I_{e x}-I_{\text {code }}\right|}{I_{e x}} \cdot 100 \%=\frac{|14.222139-14.574774|}{14.222139} \cdot 100=2.47 \%
$$

What proves to pay more attention to this method which will replace classical Gauss one. Don't forget to note that in irregular nodal distribution case it is necessary to determine all geometrical area characteristics in the beginning stage and then to proceed to integral calculation. 


\section{References}

Books:

[1] Rong Liu, Meshfree methods: moving beyond the finite element method (CRC Press LLC, 2003).

Theses:

[2] Zhang Guiyong, Theory Development of Meshless Methods for three dimensional and adaptive analyses of Mechanics problems, doctoral diss., National University, SINGAPORE, 2007. 